\title{
form
}

Vol I4, No 2 (202I)

\section{Crafting Ceramics through the Use of Virtual Reality}

\begin{abstract}
This paper reflects ongoing research about how new technology creates new possibilities within crafting and how it can build on traditional techniques within the field of ceramics. The research explores how virtual reality (VR), using Oculus Rift and 3D-printing in clay is suitable to link to the process of throwing vessels within ceramic crafts. The quality of the crafted objects in question is based on the ability of the presented technique to combine throwing and pattern-making as an integrated process while building up the thrown object, here named virtual pattern-throwing. Furthermore, it is argued that this use of VR for throwing makes it possible to recall a hands-on experience and thus weave the quality of the traditional craftsmanship with the virtual within the process of making vessels.
\end{abstract}

Keywords:

Ceramics, Digital Crafting, Craft, 3D Clay Printing, Virtual Reality.

\section{INTRODUCTION}

This paper reflects ongoing research about how novel digital means create new interfaces and processes between humans, space and material. Advances in 3D virtual environments allow us to sculpt in realtime based on spatial hand gestures. At the same time, digital technology, such as 3D printing, allows bridging from the digital design environment to fabrication.

This research focuses on the possibilities that virtual reality (VR) opens up for ceramic craft practice. Thus, this contribution is about how new technologies create new possibilities within crafting and how it can build on and link traditional techniques. For this purpose, the research explores how VR, by using Oculus Rift and 3D printing in clay, links to the process of crafting within the field of ceramics. Furthermore, the wheel for throwing and the shape of a vessel is chosen as the starting point for exploration and experimentation. 


\section{METHOD}

The method is explorative and experimental. Experimental design practice is used as a method of inquiry and a reflective practice, in which the designer engages in a reflection through action and on the action (Schön, 1993). Design inquiries in the research are also used as a material practice and contribution to knowledge production (Brandt \& Binder, 2007; Koskinen et al., 2008), and is concerned with "moving away from the existing and the known, through intentional actions to arrive at an as yet unknown, but desired, outcome" (Downton, 2003). The means to enter and engage the unknown is a set of consecutive experiments. Design is, for this purpose, a powerful form of experimentation, a means of enquiring and producing knowledge inspired by Binder and Redström's (2006) notion of "exemplary design research":

It is "exemplary" in the sense that it enables critical dissemination primarily by creating examples of what could be done and how i.e., examples that express the possibilities of the design programme and more general suggestions about a (change to) design practice. (Binder \& Redström, 2006, p.3)

This paper intends to provide insight into these experiments and the potential they may exhibit.

\section{DIGITAL CRAFTING}

In this research, crafting is based on the idea that interaction with a responsive material guides the ceramicist (Leach, 1940; Dormer, 1994), and that crafting and execution work together in a way that is intuitive and humanistic (Leach, 1940). Following Leach and Dormer, the traditional craft can be understood through two parallel levels: its immediate interface to matter, which can provide instant feedback, and the consistency of design logic. and material processing. Instead of thinking of craft and technology as diametric positions, this paper proposes to see technology as an enabling forcefollowing McCullough's (1998) idea about a close connection between digital work and craft practice.

A novel set of design software makes it easier for artists, architects, and designers to develop tools specific to their projects. One such example is the 3D-modelling software Rhino (Robert McNeel \& Associates http://www.rhino3d.com/) and its graphic programming interface, Grasshopper, developed by David Rutten (http://www.grasshopper3d.com/).

Overall, when these tools are developed and practiced in workflows with 3D rapid prototyping it can be called digital crafting (Thomsen et al., 2012). Digital design tools no longer operate in a disembodied space of representation, which takes the designer away from matter; instead, they function to extend the designer's ability and senses to craft material.

Although this approach extends the space of design into the making of specified materials and behaviours (Thomsen et al., 2013), it might not deal with its immediate interface to matter, providing instant feedback in the very moment of giving form.

Nevertheless, experimentation with human interaction in 3D virtual environments has shown interesting results. These 3D digital environments are meant for designing a 3D form that is responsive to the movements of the body in real-time and provides instant feedback at the very moment of giving form. Such environments have shown the user's ability to provide bodily engagement as a powerful means to gain overall control within a virtually complex environment and gain access to the embodiment of skills (Hansen \& Tamke 2015, 2019). It is such human interaction and environments that are of interest for this research.

\section{VIRTUAL REALITY}

In this paper, the term VR is defined as a simulation of an imaginary world where the user can interact with the environment (Craig, 2013) through the use of a headset accompanied by two hand controllers. Oculus VR (https://www.oculus.com), a division of Facebook Inc., has developed different virtual reality headsets, including the Oculus Rift that is the object of this study.

An example of the overall setup can be seen in Image 1, which shows the headset and controllers used within the 3D virtual environment. In the virtual environment, the position of the hands 
appears as virtual hands. Such a setup is used in various contexts for different purposes and, in this study, will focus on the purpose of 3D designing and sculpting.

In this paper, Oculus Rift is explored in the 3D sculpting environment, Oculus Medium (https://www.oculus.com/medium). Oculus Medium is primarily used to sculpt 3D characters for a virtual environment but is also used for designing objects for 3D printing on an experimental basis. Nevertheless, similar 3D environments for VR, such as Gravity Sketch (https://www.gravitysketch.com), are developed and explored in a related field such as ceramics. One example is an experimental project about virtual hand-knotting of three-dimensional objects by textile designer Nithikul Nimkulrat in collaboration with Aaron Oussoren (Nimkulrat et al., 2019). Furthermore, the use of VR within industrial design and engineering are well explored e.g. for idea ideation (Ekströmer, 2019), for the automotive industry e.g. by Vector Suite (https://www.vectorsuite.co.uk/) and for developing automotive lighting design by game engines (Ekströmer et al., 2019).

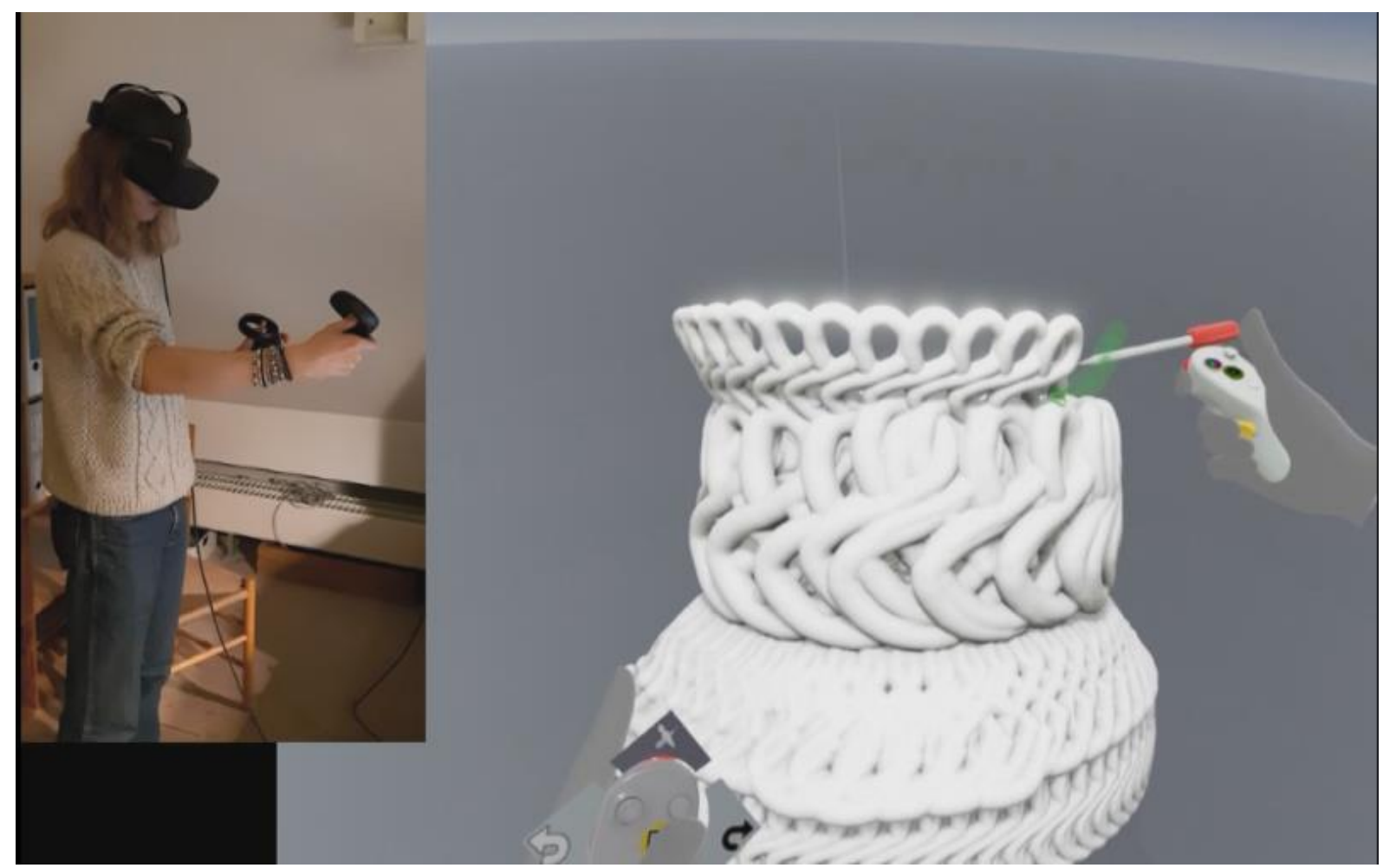

IMAGE 1. An example of the overall setup that shows the headset and controllers in use and the 3D virtual environment. In the virtual environment, the positions of the hands appear as virtual hands with controllers.

\section{SCULPTING IN OCULUS MEDIUM}

The process in this experiment is based on a two-step process within the 3D virtual sculpting environment Oculus Medium. The first step entails developing a tool for using a virtual wheel. Here named the wheel-tool. The second step is the actual sculpting of the vessel through the use of the wheel.

The wheel-tool is developed by drawing it with a brush in Oculus Medium (see Image 2). This makes it possible to draw a 3D shape (see Image 3) that, in this case, becomes the wheel-tool for using the wheel (see Image 4). The workflow with the same wheel-tool can be utilised based on scale, angle and speed (see Image 5, 6, and 7), which is reflected in the final object in the form of a dynamic and varied pattern on the surface of the rotated object (see Image 8). 


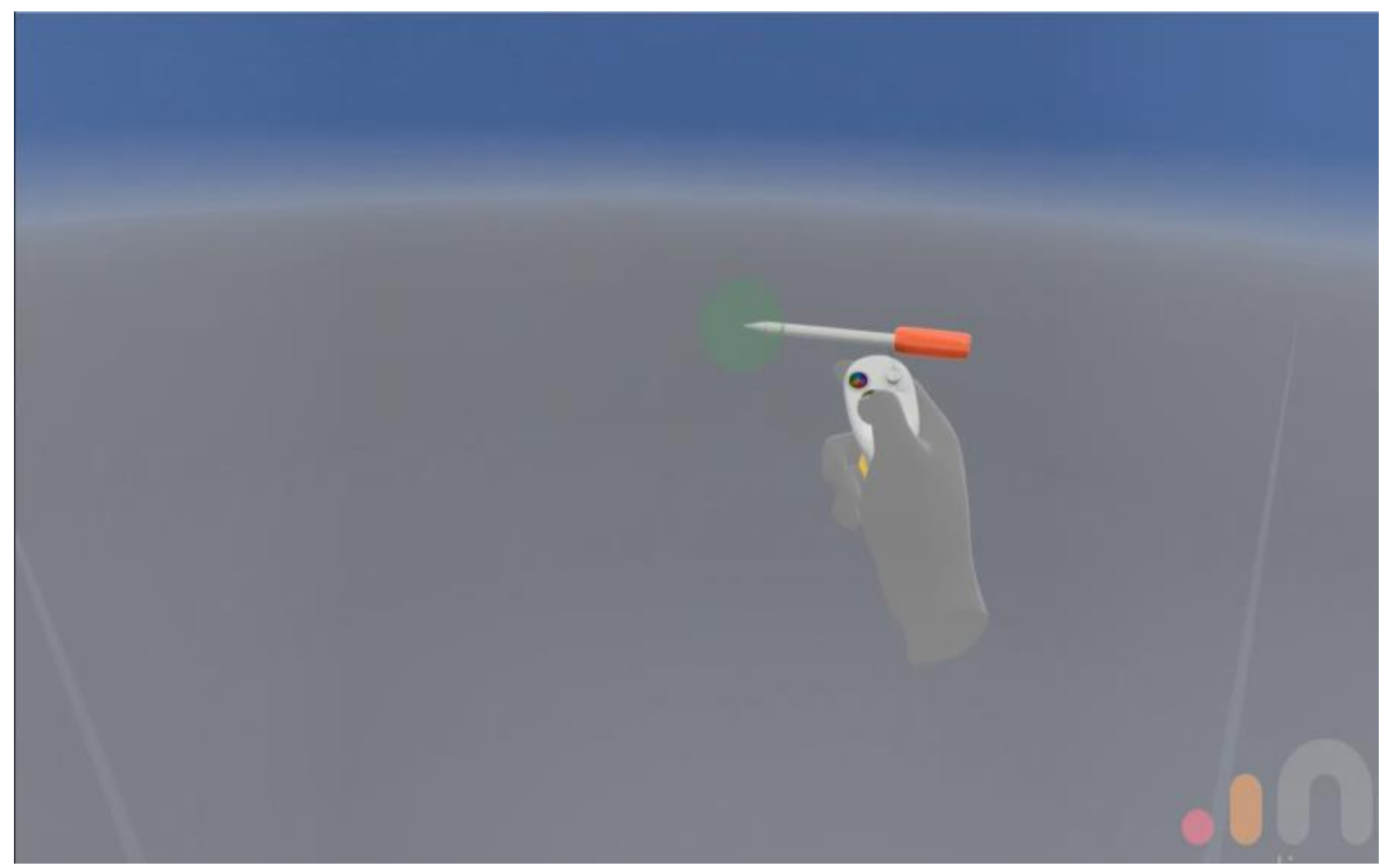

IMAGE 2. The wheel-tool is developed by drawing it with a brush in Oculus Medium

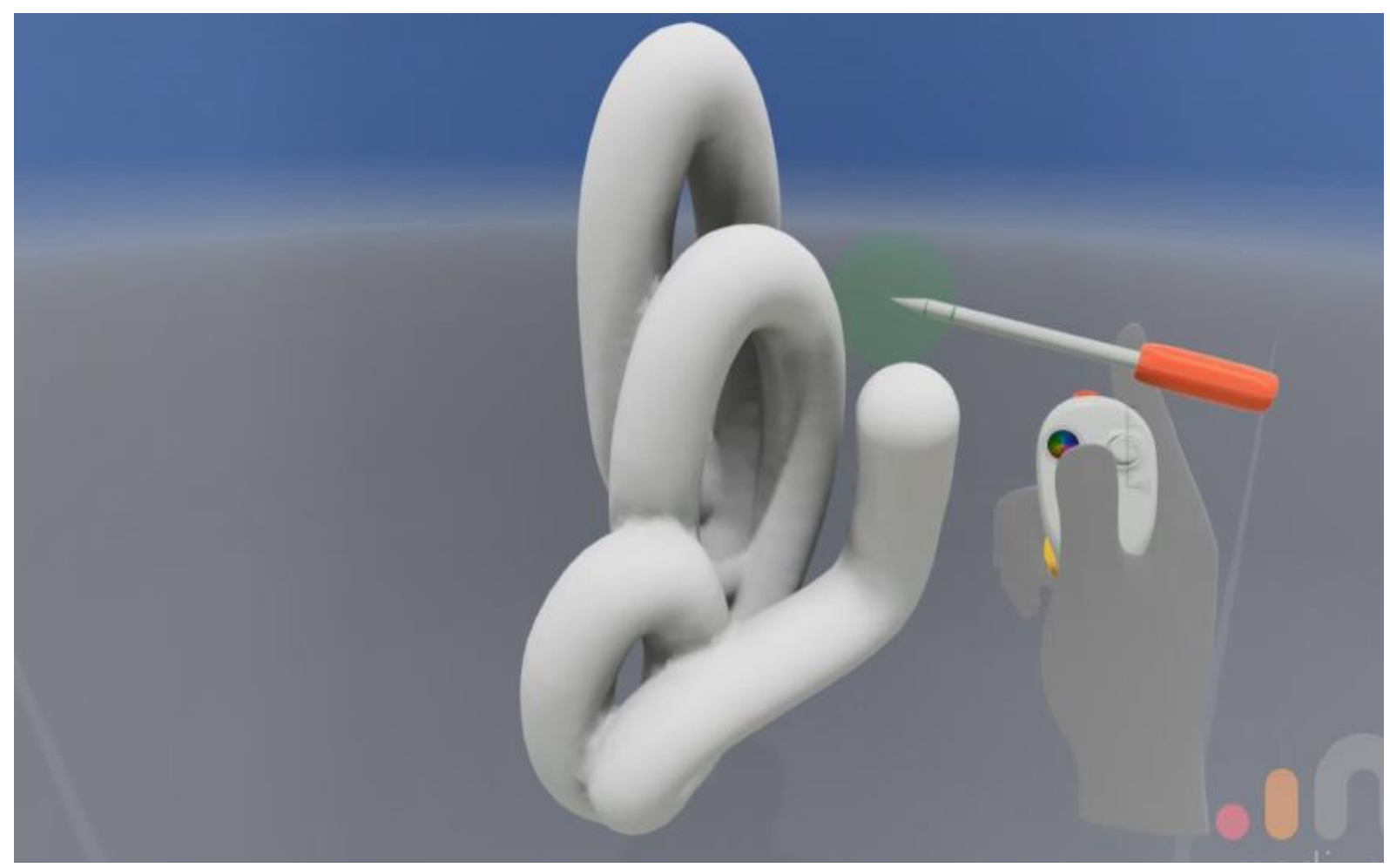

IMAGE 3. Drawing a 3D shape that becomes the wheel-tool. 


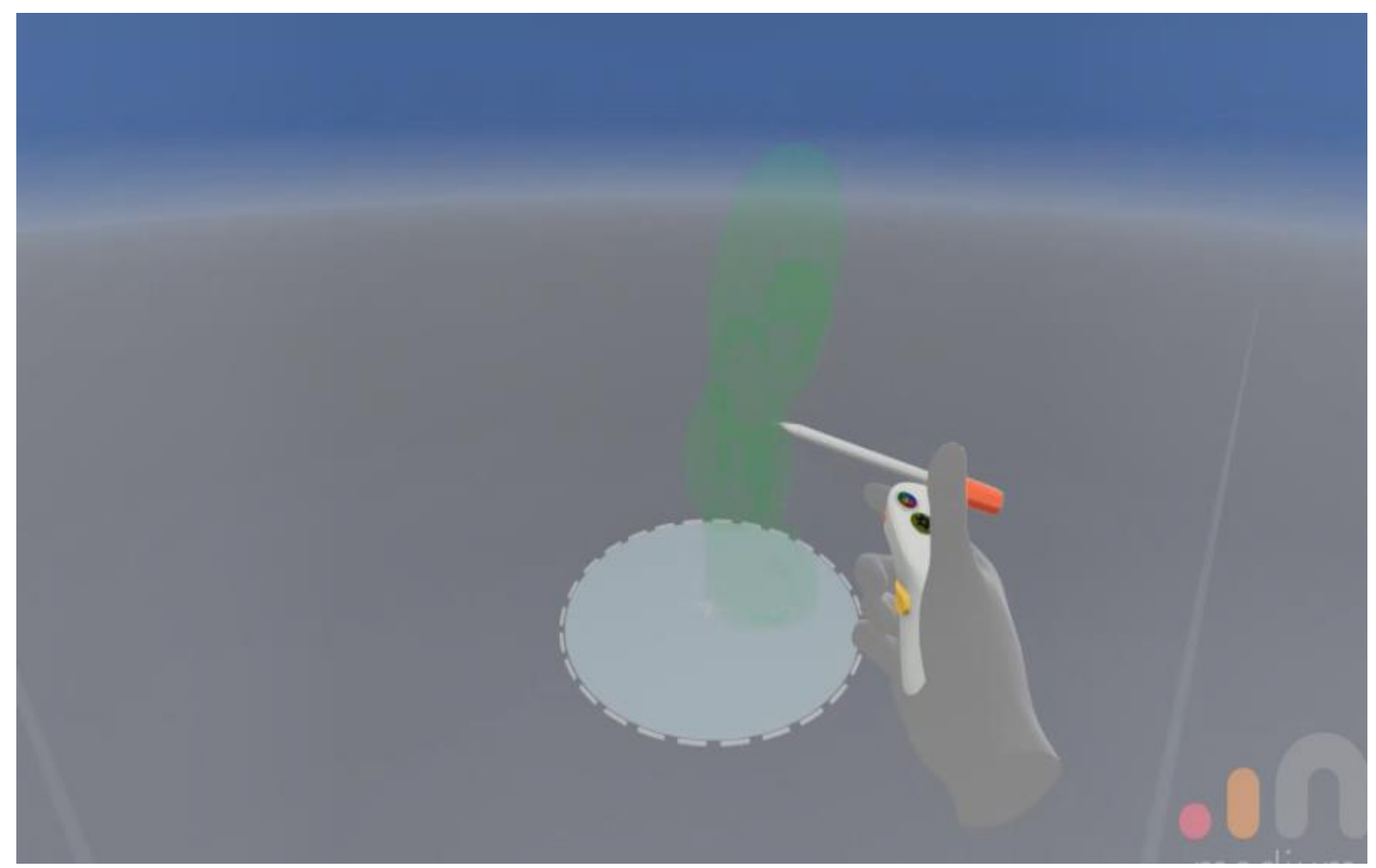

IMAGE 4. The wheel and the wheel-tool.

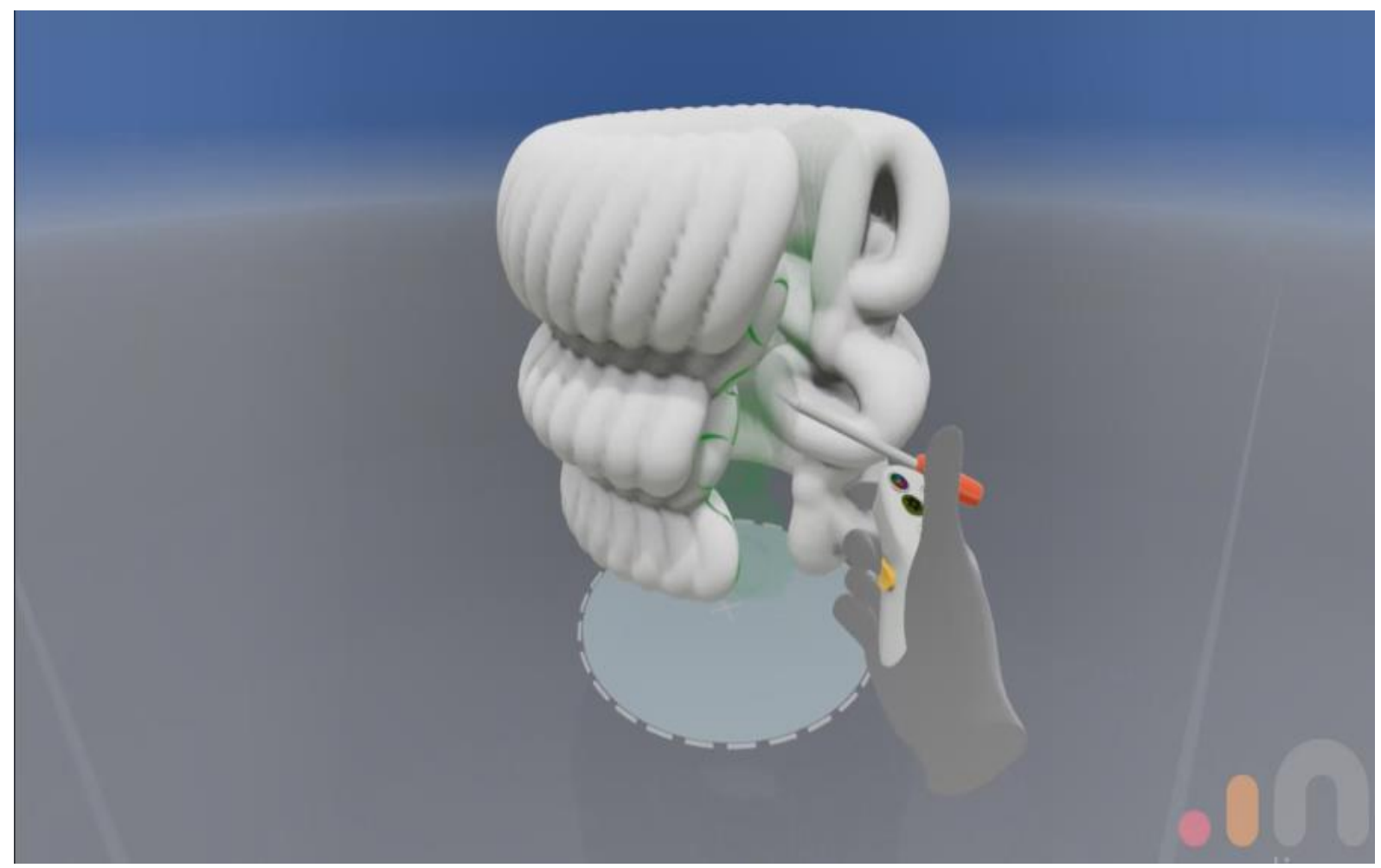

IMAGE 5. The workflow with the same wheel-tool can be utilised based on scale, angle, and speed. Example 1. 


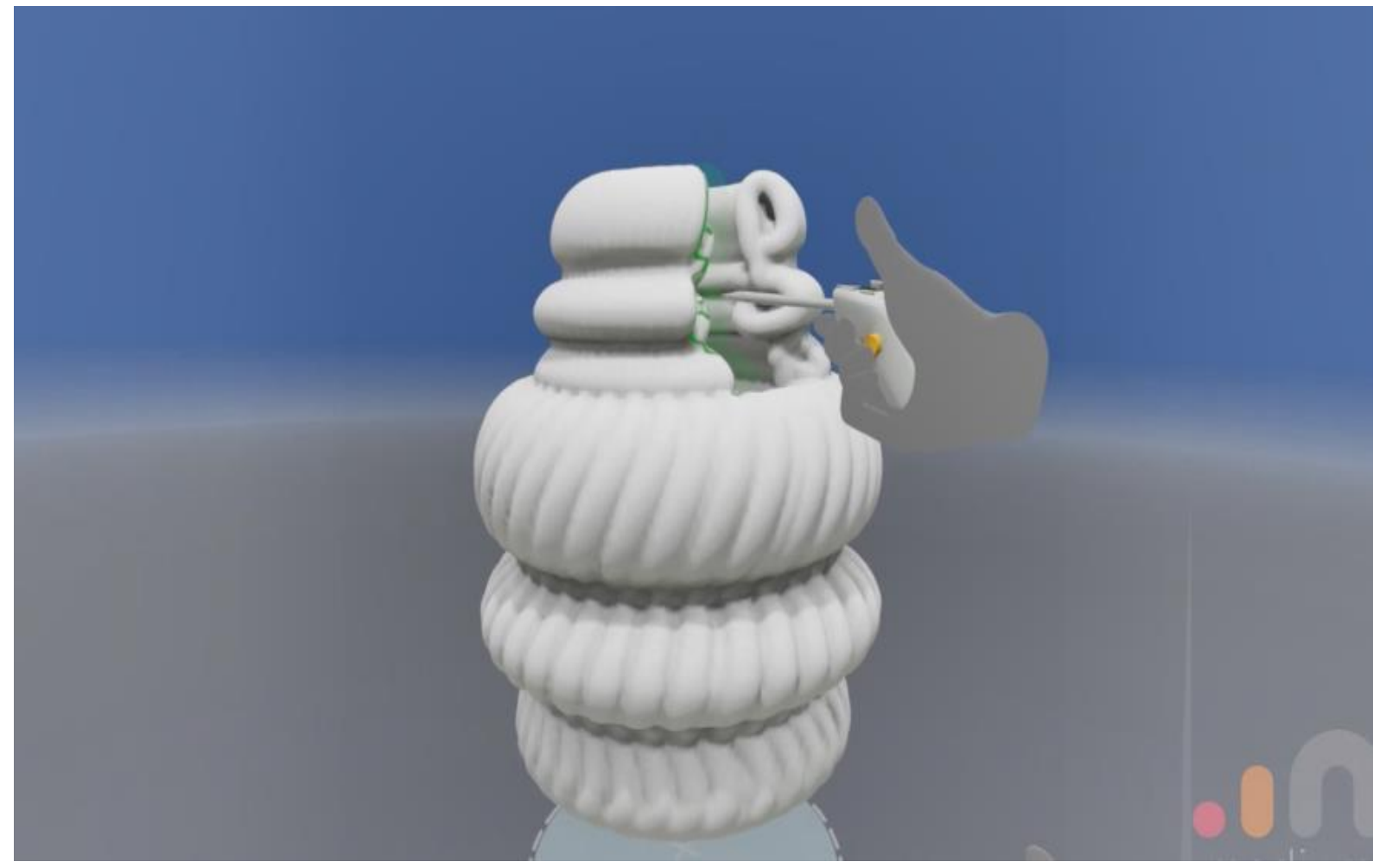

IMAGE 6. The workflow with the same wheel-tool can be utilised based on scale, angle, and speed. Example 2.

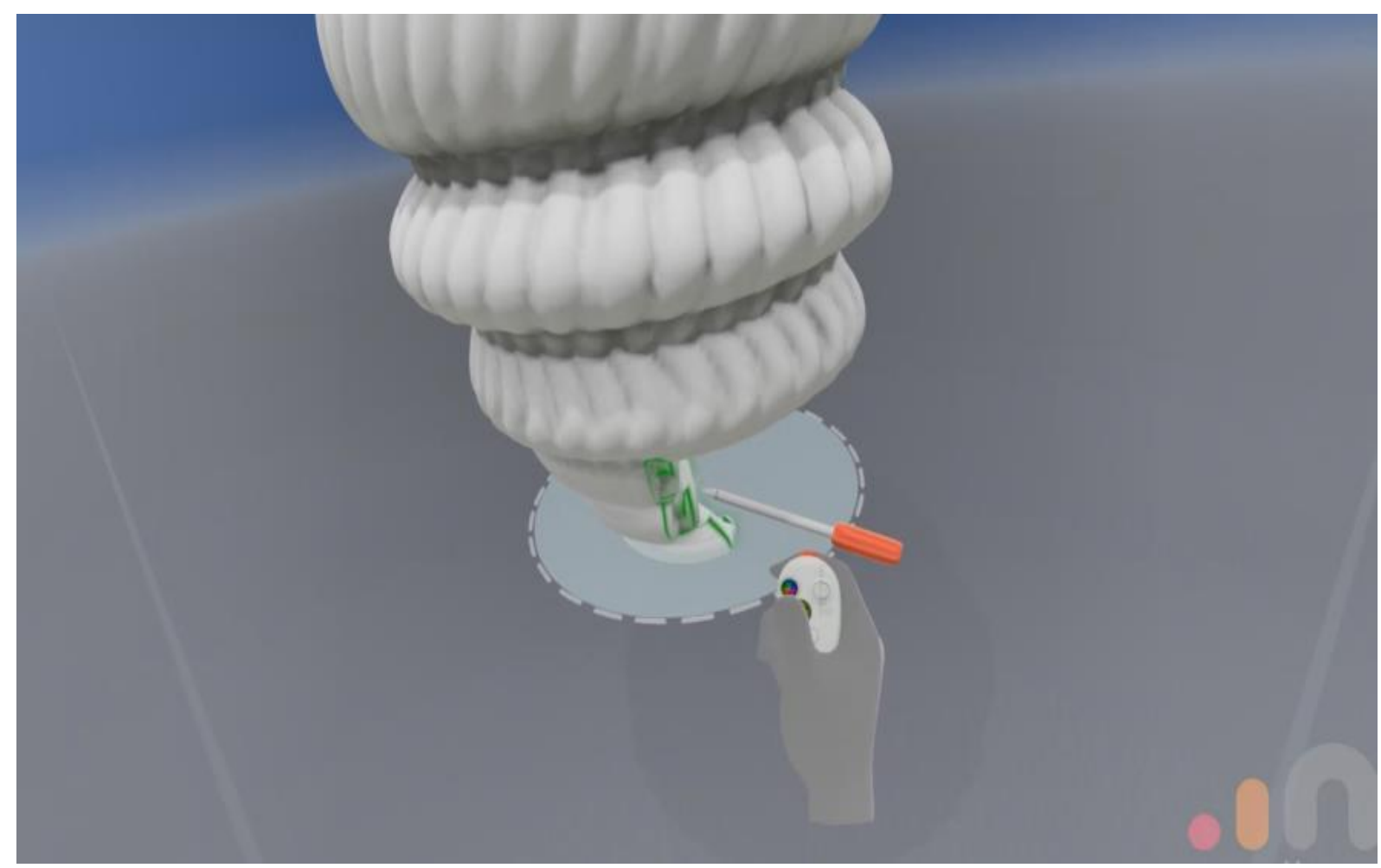

IMAGE 7. The workflow with the same wheel-tool can be utilised based on scale, angle, and speed. Example 3. 


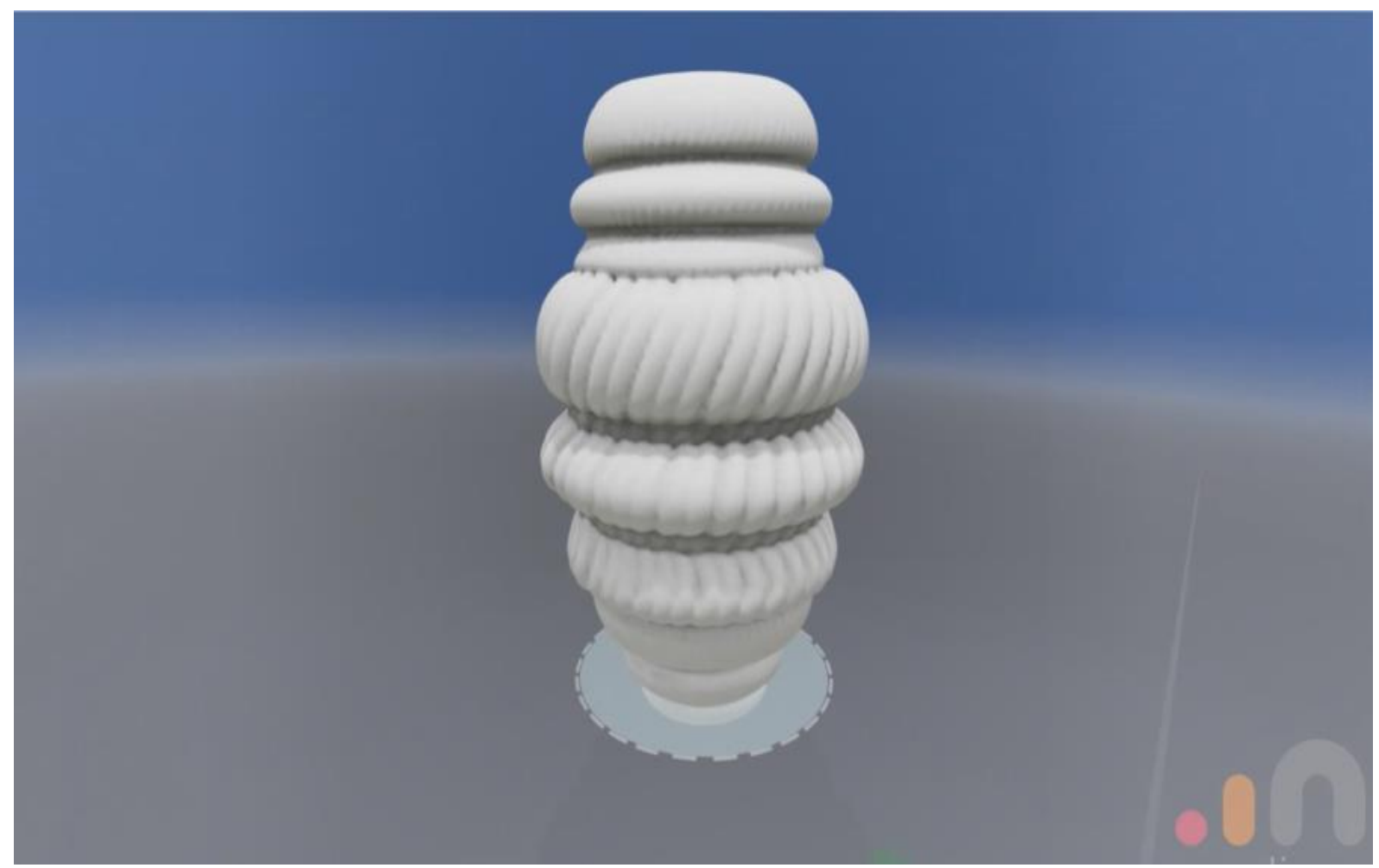

IMAGE 8. The final object with a varied pattern on the surface reflects the workflow with the wheel-tool.

Finally, to make a ceramic product, the digital form is 3D printed in clay. In this case, through the use of a WASP 40100 (see Image 9). The transformation from a digital to physical form depends on the 3D printing techniques and resolution. In this case, the print layer is emphasised by a layer height of $2 \mathrm{~mm}$. This makes the clear horizontal lines contrast to the pattern's vertical direction based on the wheel-tool (see Image 10).

\section{EXPLORATION OF PATTERN AND CONSTRUCTION}

The exploration of the pattern became the pivotal point for this research.

An important observation was a minor gap within the pattern on one of the first printed objects (see Image 11). This observation opened up exploration of the cross border between the pattern and the construction of the body.

For that purpose, an earlier observation of the relation between speed and pattern was of great importance. A faster speed makes the shape of the wheel-tool more visible. Thus, the speed of the virtual wheel was increased until the shape of the wheel-tool appeared as single elements in certain areas of the object (see Image 12). These single elements turned the pattern-making into a kind of ornamental construction of the vessel.

Several tests were developed in the 3D environment, ranging from simpler (see Image 12b) to more complex constructions (see Image 12c). These objects are characterised by central areas with open walls constructed by 3D ornaments and simple areas with a top and bottom pattern. 

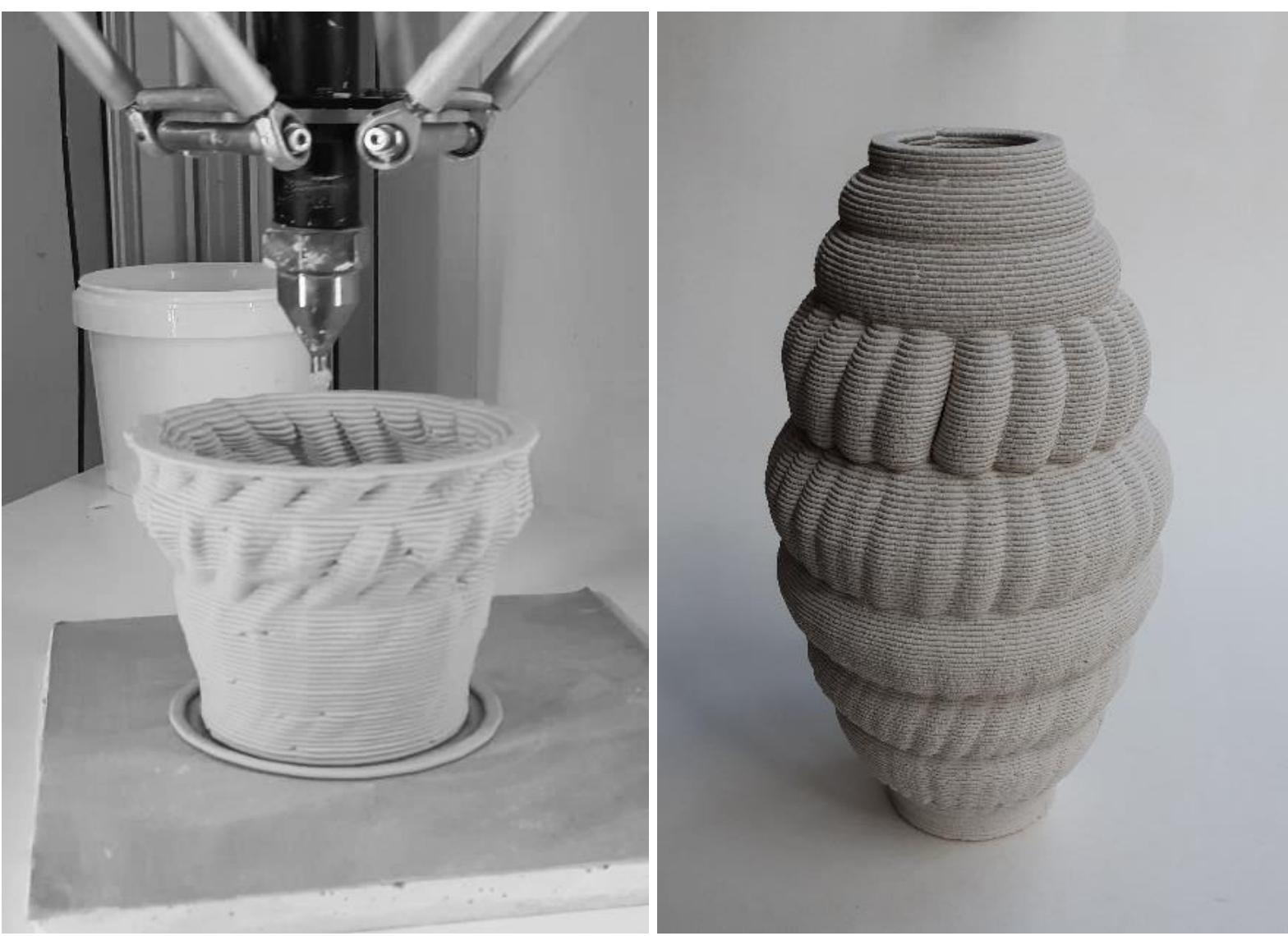

IMAGE 9 AND 10. The digital form is 3D printed in clay through the use of a WASP 40100 (Image 9). A layer height of $2 \mathrm{~mm}$ makes clear horizontal lines as a contrast to the vertical direction of the pattern based on the tool (Image 10).

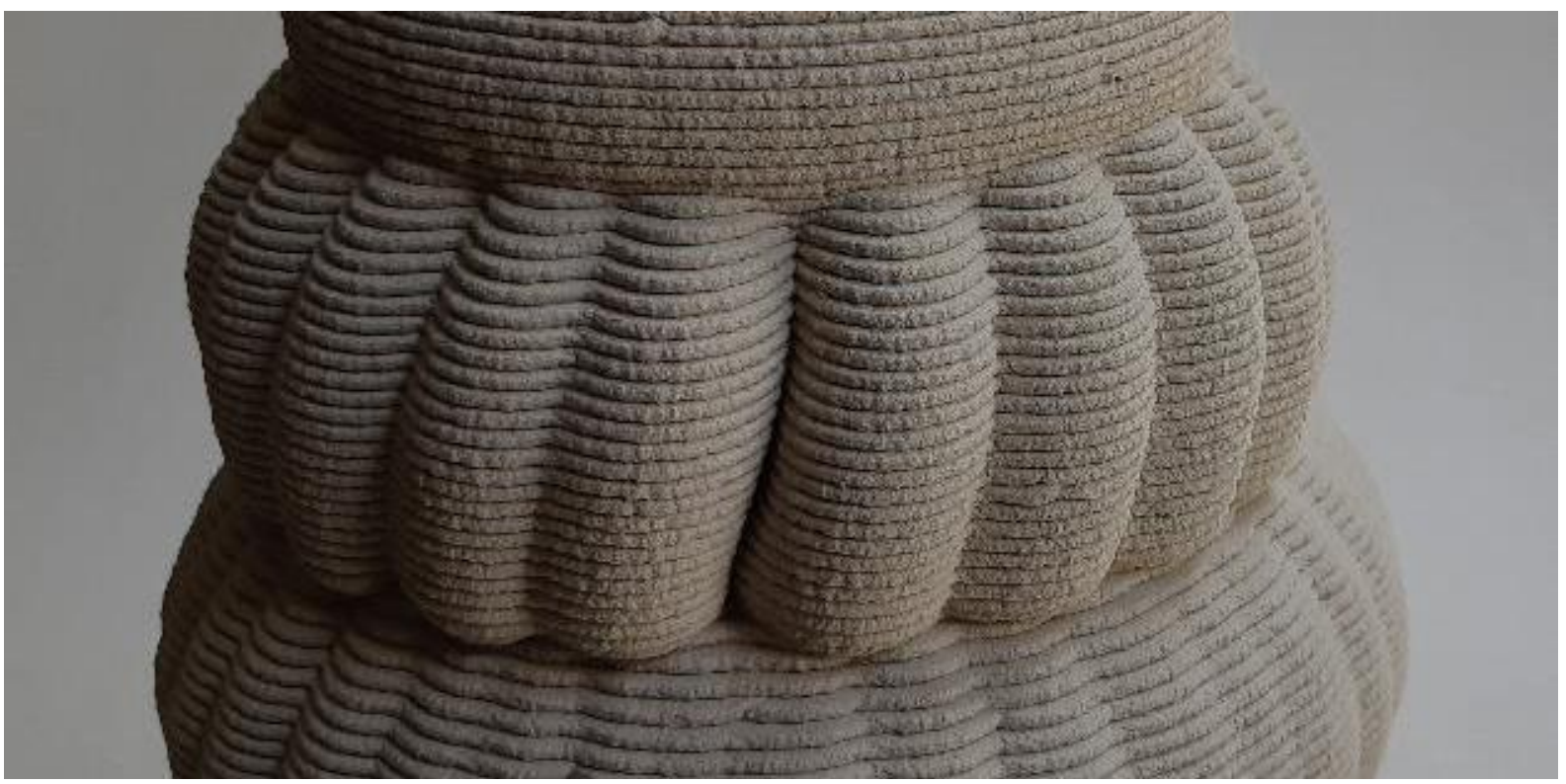

IMAGE 11. The observation of a minor vertical gap in the pattern opened up exploration of the cross border between the pattern and the construction of the body. 

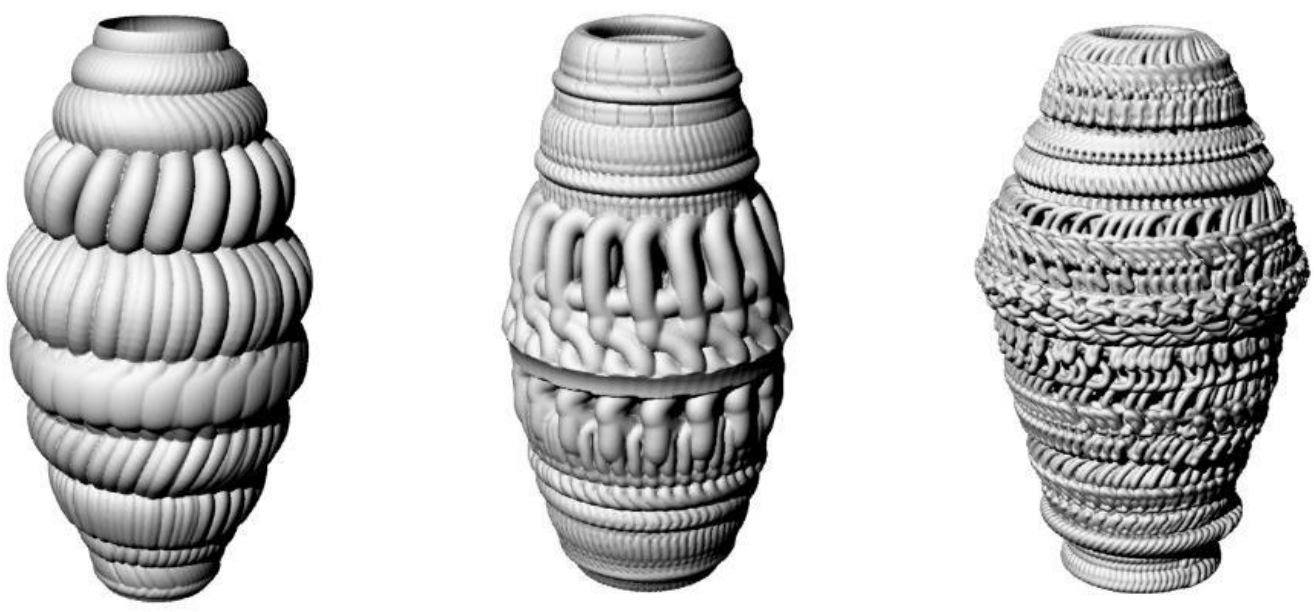

IMAGE 12. A; B; C: The speed of the virtual wheel was increased until the tool appeared as single elements in certain areas of the objects.

\section{EXPLORING THE WALL CONSTRUCTION}

Since the process of 3D printing changes the expression of the digital object, it was important to explore the possible quality of the shape when it was printed. The objects on Image $12 \mathrm{~b}$ and $12 \mathrm{c}$ were explored to test the balance between the resolution of the 3D printing and the complexity of the ornamentation. The object on Image 12c was too detailed for 3D printing since the resolution of the 3D printer nozzle was larger than the pattern details. Nevertheless, print size and resolution of the 3D printer nozzle is interdependent and the conclusion was in this case based on the decision of a print height of $40 \mathrm{~cm}$. Thus, it was decided to further experiment with $3 \mathrm{~d}$ printing the object on Image $12 \mathrm{~b}$.

As part of the testing, a partial print of the object on Image 12b was executed (see Image 13 and 14). While 3D printing, the double-sided wall around the open area in the centre of the object showed a new possible area of interest. This area of interest was the double-sided open walls since the inside and outside of the perforated construction area became equal and appeared as a 3D folded pattern-like surface. This was further explored and unfolded as close-up prints (see Images 15, 16 and 17).
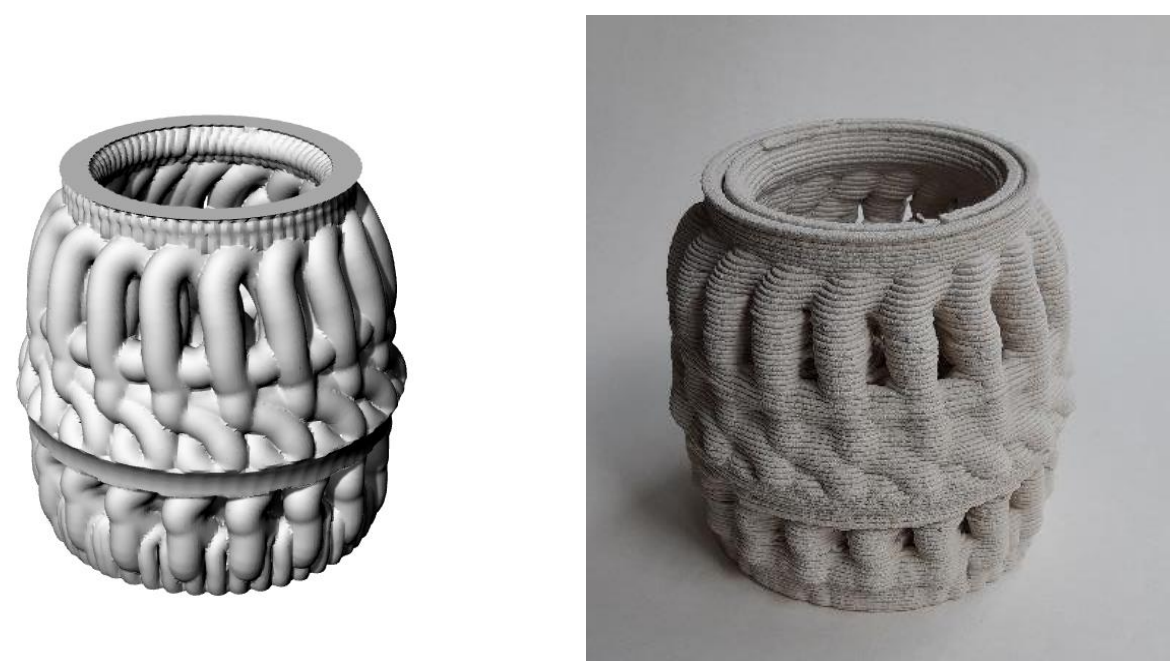

IMAGE 13 AND 14. The 3D digital object chosen for further exploration by a partial 3D print in clay (Image 13). The partial 3D clay print of the main open part of the object (Image 14). A central step towards a new area of investigation of the doublesided open walls. 


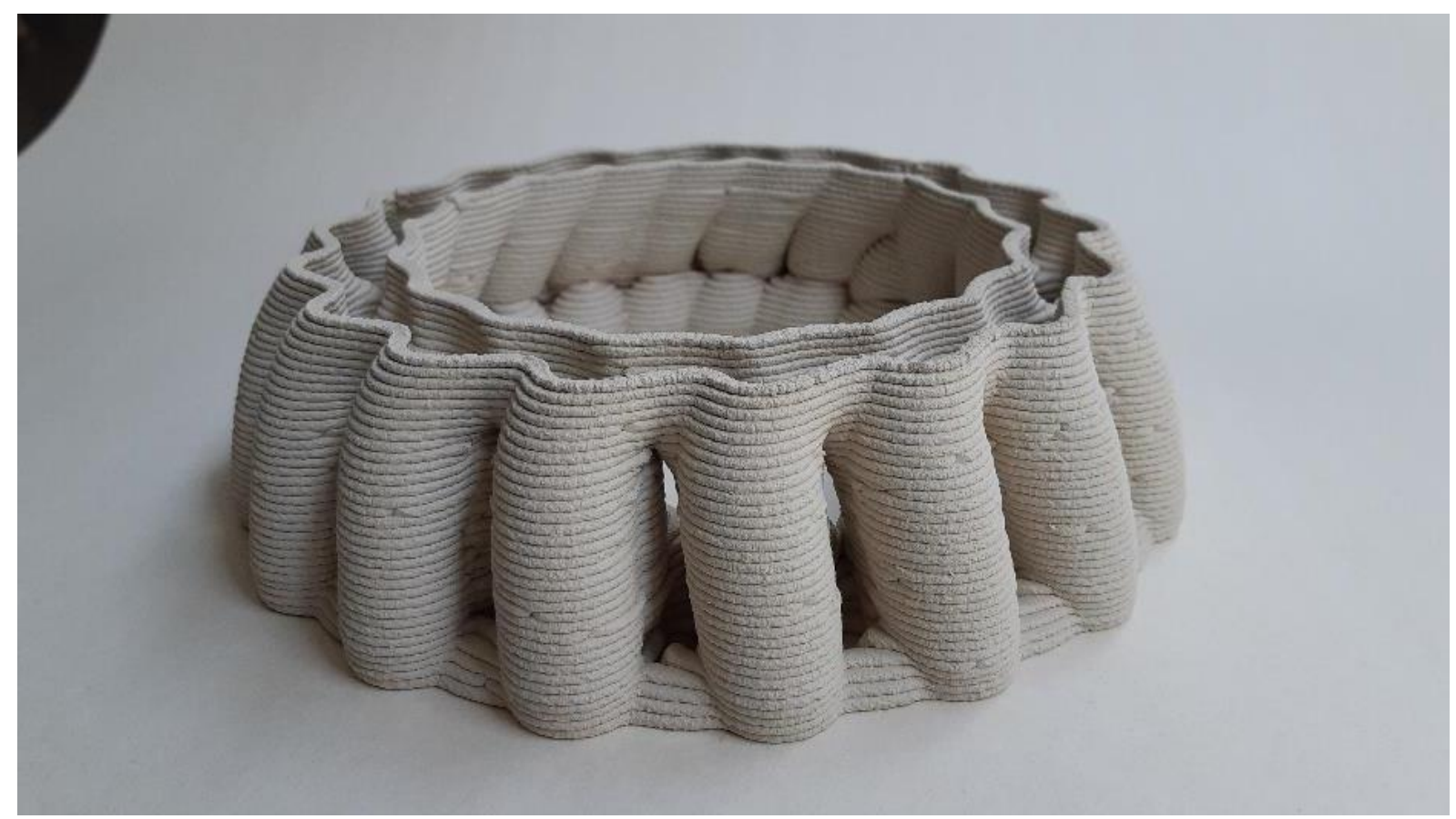

IMAGE 15. Close-up 3D print of the centre of the object. The inside of the walls became a central part of the ornamental expression because of the double-sided open walls.

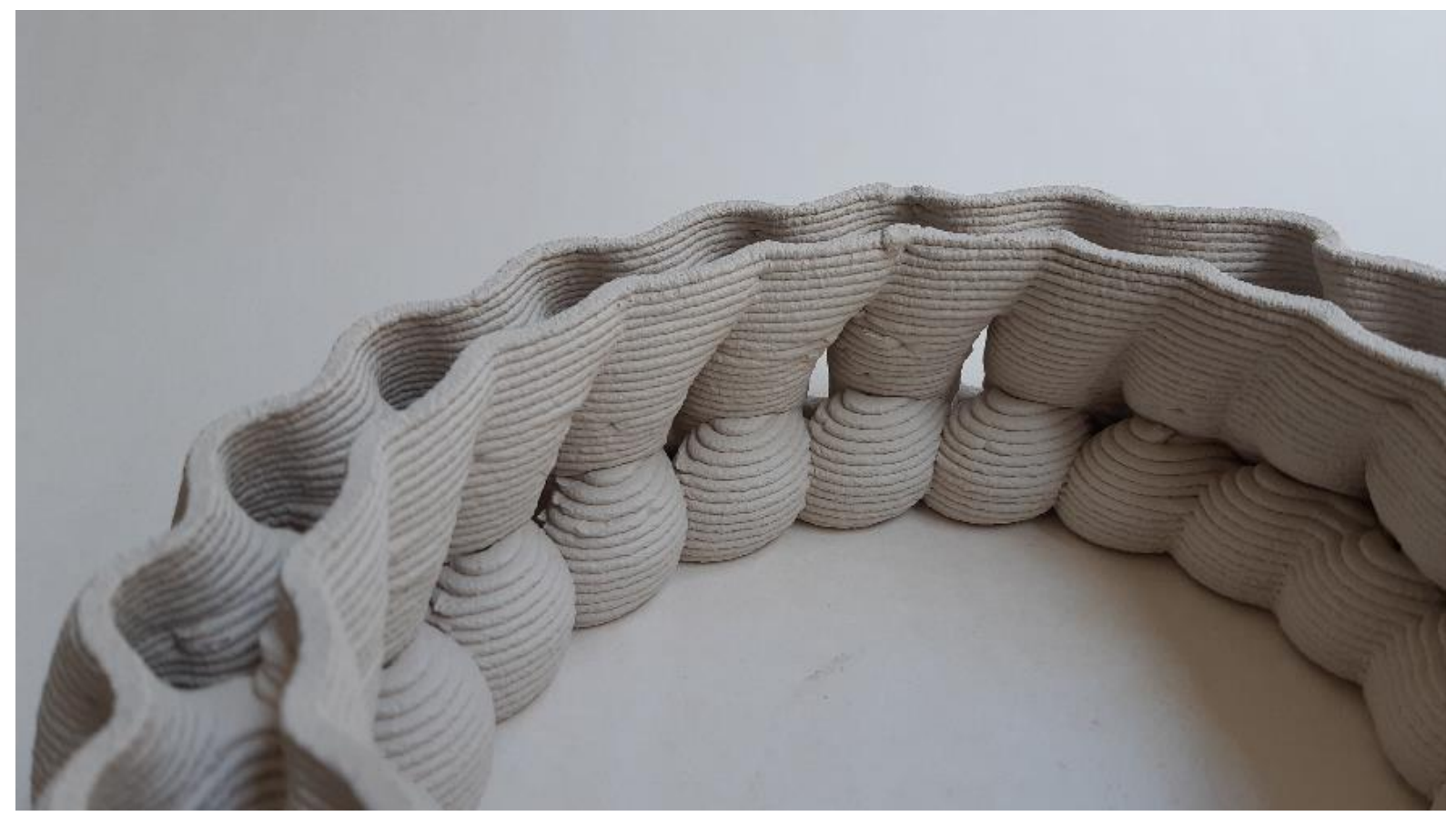

IMAGE 16. Detail of the close-up 3D print from Image 15. 


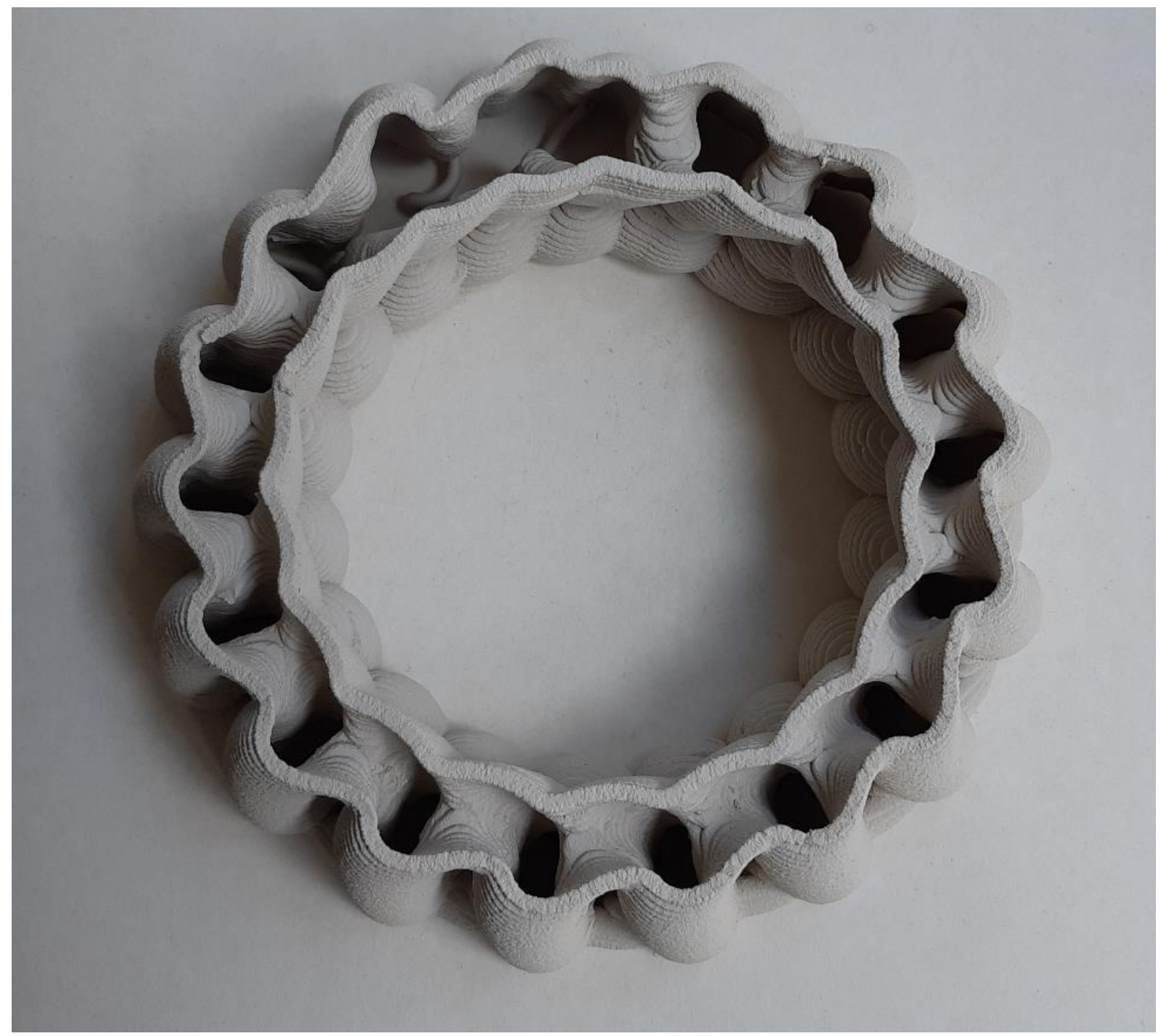

IMAGE 17. Top-view of the close-up 3D print from Image 15.

\section{REFLECTION AND DISCUSSION}

Focusing on ceramic practices, the question was how and where traditional craft-based knowledge, rooted in skills and experience of making three-dimensional objects, can meet and inform novel ceramic processes that utilise VR. Moreover, how such digital tools and means aid craft research was also be considered.

These questions have been explored through experiments using Oculus Rift in the 3D sculpting environment Oculus Medium. The shape of a vessel was chosen as a frame for exploration and experimentation. The first exploration of VR by Oculus Medium identified the virtual wheel as a tool and common ground for experimentation because of its similarity with the traditional wheel for throwing clay.

\section{Similarities}

During the exploration of the virtual wheel, a drawn 3D shape was used to build up a vessel-like shape. The technique is named pattern-throwing. The quality of crafting by this technique is based on its ability to combine throwing and making patterns while building up the vessel. In general, these patterns are characterised by vertical ornamental shapes that makes up a relief. These vertical ornamental shapes differ from the pattern of the horizontal lines seen from the 3D clay print technique and the horizontal grooves coming from fingers or tools when throwing in clay. 
Several experiments were completed by this technique, and interesting references to traditional craftbased knowledge, rooted in skills and experience, have been identified.

The first result of the pattern-throwing, here named a pattern-throwing level 1, resulted in a vessel-like shape decorated with a relief pattern as an integrated part of the surface (see Image 10). The result was notable since it clearly references the traditional techniques of impressing by carving or using stamps into soft clay by pressing different shaped objects (Mussi, 2020).

Subsequently, the technique was developed to another level, here named pattern-throwing level 2, by increasing the speed of the VR wheel for throwing. The quality of crafting by this technique was found in its ability to construct an open, ornamental wall construction that was built up simultaneously with the vessel (see Image 13). The result of the perforated wall is notable since it has a clear reference to the traditional techniques of piercing the wall of a ceramic artefact. Pierced decoration refers to holes cut into jars and plates as a decoration by the use of sharp-bladed tools and boring hole implements (Mussi, 2020).

Finally, a partial 3D clay print based on the techniques of pattern-throwing level 2 opened up new possibilities, here named pattern-throwing level 3 . The perforated area was opened up, and the inside of the double-sided wall became visible (see Image 15, 16 and 17). This was notable since the inside and outside of the perforated construction area became equal and appeared as a 3D folded pattern-like surface. The folded pattern-like surface is notable since it references what might happen when the wall becomes too thin when throwing clay. The wall starts folding either by purpose or by accident. A technique that, among others, has been well expressed and explored by the art potter George Ohr (Hecht, 2013).

Common to all these experiments with pattern-throwing is the strength of combining patterns and throwing as a whole in the process of making a vessel. Initially, this idea was not based on a traditional technique within ceramics, but similarities were found throughout the process of making, which inspired and affected the experimentation. Nevertheless, the process of making is quite different depending on whether it is fulfilled by the VR tool or by traditional techniques.

\section{Differences}

To obtain the above-mentioned expression that weaves the form and the ornamental pattern by traditional techniques, e.g., by carving, stamping and perforating a thrown vessel, is typically based on a two-step process. First, the vessel is thrown in clay, and second, the carving or piercing of the vessel is fulfilled. This makes the thrown vessel the base for expression.

The primary difference is the process of pattern-throwing by using VR obtained by a one-step process that combines throwing and making ornamental patterns while building up the vessel. This is important since it makes the pattern-making and throwing happen as an integrated process. Pattern and throwing work as a whole, enabling the pattern to serve as the base for the expression.

Nevertheless, by pattern-throwing, we only deal with a virtual representation of the vessel and not the actual making in clay. This happens by 3D printing in clay as a second step. At first, this seems to differ from the idea that the responsive material guides the ceramicist (Leach, 1940; Dormer, 1994). However, upon closer inspection, it opens up new possibilities that arguably are based on the responsive material's role. First, 3D printing in clay relies on experiential knowledge that has to be embedded when designing in VR. For example, when the 3D printer builds up the wall of the vessel in clay, the experiential knowledge that is concerned with the plasticity of clay is crucial. If the vessel is too curved, the vessel will collapse. Second, the ceramist can improvise and intervene while 3D printing by changing the speed and flow of the clay extrusion (Hansen and Falin, 2016). This use of the printer enables a wide range of possibilities concerned with the role of the responsive material. This is out of range within this research and relies on experiential knowledge that deals with instant feedback in the very moment of giving form.

\section{Hands-on Experience in Virtual Reality}

The actual making of a digital form by VR is not bound with physicality and by touch as when throwing in clay. When throwing in clay, the ceramicist can explore and make with an immediate interface to matter. The interplay between hand, clay, and the wheel as the tool is central and intuitive. Thus, 
throwing clay is largely a tactile skill (Groth et al., 2013). This is important and differs fundamentally from virtual pattern-throwing that is without friction and physicality. Nevertheless, VR offers the same sense of space as with the physical object (Ekströmer et al., 2019) and allows us to be present in this case by our hands. Textile designer Nithukul Nimkulrat describes how "drawing in VR recalled hands-on experience" (Nimkulrat et al., 2019) and enhanced her "understanding of the positions of strands of knots in a three-dimensional space" (Nimkulrat et al., 2019).

These aspects are notable since they differ from the experience of using 3D software on a typical computer screen, such as the 3D modelling software Rhino. When crafting by virtual pattern-throwing, the sense of space and form felt present regarding human interaction. It was even easy to explore and experience the same form in different sizes in relation to the body, e.g., from hand size to the size of a house, which extends the way of exploring new possible scenarios for the object. While we do not deal with the tactile aspect within VR, the use of VR enables the user to recall hands-on experience to a certain degree because we sense the form and space as in the real world. Nonetheless, it is a significant advantage for the craftsperson using VR to be well experienced within the field of craft in question because they are able to recall their hands-on experience and realise the final object in the right material.

\section{Metamorphosis}

When we as craftspeople discover a new tool, we become curious. Our method is explorative and experimental and is used for inquiry and reflective practice (Schön, 1993). We ask what can be done and how and are driven by experiments (Binder and Redström 2006). The tool is explored and not necessarily what it is intended for. It is about the craftsmanship of risk and not of certainty (Pye,1968). The exploration of the three levels of pattern-throwing by Oculus Medium showed similarities with traditional techniques within throwing clay and allowed rethinking of crafting within the fields of ceramics. The use of VR has even extended our way of crafting and created new possibilities.

Richard Sennett (2008) writes about metamorphosis concerning the invention of new tools. Within the field of ceramics Richard Senneth describes how the invention of the potter's wheel "suggested an entirely new way of building up form than the rope coil; now the potter could raise a wet clay lump" (Senneth, 2008). Similarly, VR techniques have opened up a new way of making that builds on traditional craft-based knowledge rooted in skills. In this way, digital tools and means have aided this craft research. 


\section{REFERENCES}

Binder, T, \& Redström, J (2006) Exemplary Design Research. In K. Friedman, T. Love, E. Côrte-Real \& C. Rust. Wonderground 2006 Design Research Society. International Conference in Lisbon. IADE. http://unidcom.iade.pt/drs2006/wonderground/proceedings/fullpapers/DRS2006_0152.pdf

Dormer, P. (1994). The Art of the Maker. Thames and Hudson.

Downton, P. (2003). Design Research. RMIT Press.

Ekströmer, P., Wever, R., Andersson, P., \& Jönsson, J. (2019) Shedding Light on Game Engines and Virtual Reality for Design Ideation. Proceedings of the Design Society 1(1). https://doi.org/10.1017/dsi.2019.206

Ekströmer, P. (2019). A first sketch of Computer Aided Ideation. Exploring CAD tools as externalization media in design ideation. [Thesis no. 1859, Division of Machine Design Department of Management and Engineering Linköping University, Linköping, Sweden]. https://doi.org/10.3384/lic.diva-162022

Groth, C., Mäkelä, M, \& Seitamaa-Hakkarainen, P. (2013). Making sense What can we learn from experts of tactile knowledge? FormAkademisk, 6(2), Art. 2. https://doi.org/10.7577/formakademisk.656

Hansen, F. T., \& Falin, P. (2016). 3D Printing as a Ceramic Craft Tool in Its Own Right. In M. Mäkelä, B. Schmidt, P. Falin and M. Juolahti (Eds.), Ceramics and its Dimensions: Shaping the Future (pp. 114-128). Aalto University.

Hecht, E., (2013). George Ohr, The greatest Art Potter on Earth. Skira Rizzoli Publications.

Koskinen, I., Binder, T., \& Redström, J (2008), Lab, Field, Gallery and Beyond, Artifact, 2(1). https://doi.org/10.1080/17493460802303333

Leach, B. (1940). A Potter's Book. Faber and Faber.

McCullough, M. (1998). Abstracting Craft. The Practiced Digital Hand. MIT Press.

Mussi, S., (2020). Ceramic - Pottery Dictionary. http://ceramicdictionary.com/

Nimkulrat, N., Oussoren, A., Fraser, H. D., \& Doyle, K. (2019) Collaborative craft through digital fabrication and virtual reality. In RTD2019 Method \& Critique - Frictions and Shifts in RtD, March 19-23, 2019, Delft, The Netherlands. http://openresearch.ocadu.ca/id/eprint/2658/1/PUBLISHED-RTD2019_article\%2016.pdf

Pye, D. (1968). The Nature and Art of Workmanship. Cambridge University Press.

Schön, D. (1983) The Reflective Practitioner. How professionals think in action. Temple Smith.

Sennett, R. (2008). The Craftsman. Penguin Books

Thomsen, M., \& Tamke, M., (2013). Computing and Material. In R. Armstrong \& F. Simone. Unconventional Computing, ACADIA 2013: Design Methods for Adaptive Architecture. (First edition) (pp. 148-151). Riverside Architectural Press.

Thomsen, M., Tamke, M., \& Pedersen, C. (2012). Digital Crafting, A network on Computation and Craft in Architecture, Engineering and Design. http://www.digitalcrafting.dk/ 